\title{
Identification of oxidative stress-induced gene expression profiles in cavernosal endothelial cells
}

\author{
$\mathrm{CHAO} \mathrm{HU}^{1}$, YIN-YING DONG ${ }^{2},{\mathrm{YE}-\mathrm{HAO} \text { DONG }^{1}, \text { JIE-FENG CUI }^{2} \text { and JI-CAN DAI }}^{1}$ \\ ${ }^{1}$ Department of Urology, Affiliated Ren Ji Hospital, School of Medicine, Shanghai Jiao Tong University, \\ Shanghai 200001; ${ }^{2}$ Department of Oncology, Institute of Liver Cancer, Affiliated \\ Zhongshan Hospital, Fudan University, Shanghai 200032, P.R. China
}

Received February 20, 2014; Accepted November 5, 2014

DOI: $10.3892 / \mathrm{mmr} .2014 .3112$

\begin{abstract}
The aim of the present study was to explore the regulation status of genes in oxidative stress (OS)-induced endothelial dysfunction and to elucidate the mechanism of action of OS-associated genes, which induce cavernosal endothelial dysfunction in erectile dysfunction (ED). OS was established in purified cavernosal endothelial cells (CECs) using xanthine/xanthine oxidase and the differentially expressed OS-associated genes were analyzed using gene microarrays. In addition, an ED rat model was established through bilateral internal iliac artery ligation with hyperlipidemia and was verified by an intracavernosal pressure test. The selected OS-associated genes were validated in the CECs and ED rat model using reverse transcription-quantitative polymerase chain reaction. Student's t-test and one-way analysis of variance were performed using SBC analysis system. Gene microarray analysis revealed that $13090(31.92 \%)$ genes were expressed in the control group, whereas 12039 (29.35\%) genes were expressed in the treated group. The cut-off value for differential expression was set at 2.0 fold-change and 2480 genes were found to be differentially expressed compared with the control group. Of these cells, 1454 were upregulated and 1026 were downregulated. Cluster analysis identified relevant cell signaling pathways that were hypothesized to be significant in OS-associated endothelial dysfunction, including the cytokine-cytokine receptor interactions, nitrogen metabolism, coagulation cascades and cell adherens. Cxcl12, Tgfbrl, Asns, Bdkrb1 and Cdh3 genes showed a corresponding variation in the CECs and ED rat model compared with the results of the gene microarray analysis. In conclusion, in the present study, the network of differentially expressed genes and OS-associated signaling pathways
\end{abstract}

Correspondence to: Dr Ji-Can Dai, Department of Urology, Affiliated Ren Ji Hospital, School of Medicine, Shanghai Jiao Tong University, 145 Middle Shandong Road, Huangpu, Shanghai 200001, P.R. China

E-mail: jican_dai@aliyun.com

Key words: oxidative stress, microarray analysis, endothelial cell, corpus cavernosum, rats identified using gene microarray analysis were validated in the CECs and ED rat model. The results indicated that OS may lead to endothelial dysfunction through certain cell signaling pathways, inducing ED. However, further functional verification is required in order to elucidate the underlying mechanisms of OS-associated cell signaling pathways in ED.

\section{Introduction}

The majority of erectile dysfunction (ED) cases are associated with oxidative stress (OS) and occur due to certain factors that result in insufficient blood supply, including diabetes mellitus, smoking, hypercholesterolemia, hypertension and artery injury (1-3). As a prevalent physiopathological mechanism, OS increases the risk of various diseases, such as ED, in males. Previous studies have demonstrated that endothelial dysfunction, including restricted vasodilation, hemodynamic events and endothelial integrity damage, played a crucial role in the pathophysiology of ED $(4,5)$. OS was hypothesized to participate in the pathogenesis of endothelial dysfunction $(5,6)$; however, to date, studies have primarily focused on nitric oxide (NO) synthesis and reductase activity. While the mRNA expression levels of individual candidate genes have been previously reported, the map of gene regulation in OS-induced endothelial dysfunction remains to be fully elucidated (7). Microarray technology has high-throughput capability, allowing genome-wide analysis to be performed, as well as providing a less biased and more effective screening approach (8).

The aim of the present study was to investigate the regulation status of systematic genes in OS-induced endothelial dysfunction through the exposure of cavernosal endothelial cells (CECs) to xanthine/xanthine oxidase (X/XO). Differentially expressed genes were further verified in CECs, as well as the corpora cavernosa of normal and ED rats, in order to examine the effects of OS in ED. This study is fundamental for the identification of the dysregulated gene expression in OS-induced endothelial dysfunction and its implication on the development of ED.

\section{Materials and methods}

CEC preparation. The present study was approved by the ethics committee of the Animal Care and Use Committee of School of Medicine, Shanghai Jiao Tong University (Shanghai, China). 
A total of six Sprauge-Dawley rats (age, two months) were purchased from the Chinese Academy of Sciences (Beijing, China). The corpora cavernosa of ED rats were cut into $1-\mathrm{mm}^{3}$ sections and digested by sterile-filtered collagenase type II (C6885, CAS:9001-12-1; Sigma-Aldrich, St. Louis, MO, USA). The cell suspension was then cultured with endothelial cell growth medium 2 (Lonza Group, Ltd., Basal, Switzerland) at $37^{\circ} \mathrm{C}$ and $5 \% \mathrm{CO}_{2}$. Magnetic activated cell sorting (MACS; Miltenyi Biotec, Bergisch Gladbach, Germany) was used to process cells for the subsequent incubation with mouse anti-rat CD31 (Abcam, Cambridge, MA, USA) and anti-mouse immunoglobulin $\mathrm{G}$ microbeads (Miltenyi Biotec) to obtain CECs. Trypan blue (0.4\%; Sigma-Aldrich) staining for 3 min was used to detect the survival of primary cultured cells. The following two methods were used to determine the CEC purity: i) Flow cytometric analysis (FACSCalibur) with FACS Comp 3.1 software (machine and software from BD Biosciences, Franklin Lakes, NJ, USA) was performed to detect the fluorescence expression on the CEC surface. The negative control was treated with a mouse homotypic polyclonal immunoglobulin G (ab37356; Abcam, Cambridge, MA, USA) and the blank control was treated with phosphate-buffered saline; and ii) anti-von Willebrand factor (vWF; Abcam) immunofluorescence analysis was performed using a negative control to identify the CEC purity.

Primary cultured corpora CECs were divided into two groups. In treated group, OS in CECs $\left(1 \times 10^{6}\right)$ were induced using $200 \mu \mathrm{M} / 1$ xanthine (Sigma-Aldrich) and $60 \mathrm{mU} / 1$ xanthine oxidase (X/XO; Sigma-Aldrich) incubation for $48 \mathrm{~h}$. These two groups of primary cultured cells were used for microarray analysis.

CEC RNA preparation. Total RNA of CECs was extracted using TRIzol ${ }^{\circledR}$ reagent (Invitrogen Life Technologies, Carlsbad, CA, USA), according to the manufacturer's instructions, and was further purified using an RNeasy mini kit (Qiagen, Shanghai, China) and RNase-Free DNase set (Qiagen). RNA integrity numbers (RINs) were calculated using an Agilent 2100 Bioanalyzer system (Agilent Technologies, Inc., Santa Clara, CA, USA) to determine the integrity of the RNA samples.

Microarray analysis. An oligonucleotide microarray (4x44K) with $>41,000$ rat genome gene 60-mer oligonucleotides, containing the whole rat genome, was obtained from Agilent Technologies, Inc. Purified RNA was amplified and used to synthesize the first and second strands of cDNA with the primer of $\mathrm{T} 7$ oligo(dT) (Agilent Technologies, Inc.). Next, cRNA was obtained from the double-stranded cDNA using a T7 Enzyme Mix (Agilent Technologies, Inc.) and synthesized back to DNA using random primers (CapitalBio Corporation, Beijing, China) following purification. Subsequently, DNA production, from the RNAs of primary cultured cells, was labeled using cyanine dye (2'-deoxycytidine 5'-triphosphate; Agilent Technologies, Inc.) with random primers and Klenow enzyme (Agilent Technologies, Inc.). Following labeling, the treated and normal CEC samples were separately hybridized into the microarrays and incubated for $17 \mathrm{~h}$ at $42^{\circ} \mathrm{C}$. Following hybridization, the slides were washed in staining dishes (Cat no. 121; Thermo Fisher Scientific, Waltham, MA, USA) with Gene Expression Wash Buffer kit (Cat no. 5188-5327; Agilent
Technologies, Inc.) according to the manufacturer's instructions and then scanned using an Agilent microarray scanner (G2565CA; Agilent Technologies, Inc.).

During microarray scanning, the microarray scanner converted the fluorescence signal intensities into digital signals, and the signals from each spot were captured and extracted from the local background of total signal intensities. The raw data were analyzed using the Feature Extraction software 10.7 and normalized using the Gene Spring software 11.0 (Agilent Technologies, Inc.). Quantile algorithms were used to normalize the data and the uniformized data were qualified in analysis, allowing for comparisons between the microarrays. Subsequently, the genes were scored according to their normalized signal values. In order to identify the differentially expressed genes in the treated CEC group compared with the normal control group, fold-changes in the signal intensities of the genes were compared with the local background according to the following data-screening criteria: Gene signal intensities with a fold-change of $>0.5$ or $<2.0$ were omitted, while the differentially expressed genes were affirmed when the fold-change in the signal intensities of the treated CEC group compared with the normal control group was $<0.5$ or $>2.0$ (9). Next, the differentially expressed genes were further analyzed using the online SBC analysis system (SAS; version 8.0; Shanghai Biotechnologies Corporation, Shanghai, China). SAS was used to facilitate the systematic identification and categorization of differentially expressed genes into signaling pathways through enrichment analysis of individual probes representing certain genes and pathways $(10,11)$. Furthermore, SAS was used to investigate the association among the specified genes and sets of functional genes that were part of biologically relevant networks, according to a public bioinformatics databases, including the Kyoto Encyclopedia of Genes and Genomes (KEGG) (12) and the Database for Annotation, Visualization and Integrated Discovery (13), using a hypergeometric distribution to conduct statistical analysis.

ED rat model and cavernosal tissue preparation. As previously described (14), the rats in the ED group $(n=6)$ were anesthetized with intraperitoneal injection of sodium pentobarbital (30 mg/kg; Sinopharm Chemical Reagen, Shanghai, China). An incision was then made in the lower abdomen, followed by isolation and triple ligation of the bilateral internal iliac arteries, which was sustained for 12 weeks to establish the ED rat model. In order to eliminate the interruption of compensatory mechanisms for erectile function, the rats were subjected to a high-fat diet (10\% egg yolk, $8 \%$ lard, $0.2 \%$ propylthiouracil, $0.5 \%$ bile salt and $4.8 \%$ salt) for 12 weeks to induce hyperlipidemia (15). The ED $(n=6)$ and normal control rats $(n=3)$ were kept in a specific-pathogen-free environment. Evaluation of the erectile function was performed using the intracavernosal pressure (ICP) test, as previously described (16-18). The rats were sacrificed by an intraperitoneal lethal injection of sodium pentobarbital $(60 \mathrm{mg} / \mathrm{kg})$ and their corpora cavernosa were obtained and ground into powder using liquid nitrogen. Total RNA of these two groups of cavernosal tissues were extracted and used for the in vivo validation of differentially expressed genes.

Functional categories and in vivo validation of differentially expressed genes. According to the gene microarray analysis 
Table I. Sequences of primers used in reverse transcription-quantitative polymerase chain reaction.

\begin{tabular}{|c|c|c|c|c|}
\hline Genes & Primer sequences & $\begin{array}{c}\text { Melting } \\
\text { temperature } \\
\left({ }^{\circ} \mathrm{C}\right)\end{array}$ & $\begin{array}{c}\text { Annealing } \\
\text { temperature } \\
\left({ }^{\circ} \mathrm{C}\right)\end{array}$ & $\begin{array}{l}\text { Amplified } \\
\text { fragment } \\
\text { length (bp) }\end{array}$ \\
\hline Cxcl12 & $\begin{array}{l}\text { F, 5'-CATCAGTGACGGTAAGC-3' } \\
\text { R, 5'-AGGGCACAGTTTGGAG-3' }\end{array}$ & $\begin{array}{l}54.6 \\
54.1\end{array}$ & 55 & 120 \\
\hline Tgfbr1 & $\begin{array}{l}\text { F, 5'-GGCTTAGTATTCTGGG-3' } \\
\text { R, 5'-TTCTTCAACGGATGG-3' }\end{array}$ & $\begin{array}{l}51.6 \\
48.1\end{array}$ & 54.5 & 108 \\
\hline Asns & $\begin{array}{l}\text { F, 5'-AAACCAAATGGCAAAGT-3' } \\
\text { R, 5'-CTCAAAGCCTGGGAAG-3' }\end{array}$ & $\begin{array}{l}47.4 \\
54.1\end{array}$ & 54 & 111 \\
\hline Bdkrb1 & $\begin{array}{l}\text { F, 5'-CAGCCCTCTAACCGAAGC-3' } \\
\text { R, 5'-CGATACAGCAGGTCCCAGTC-3' }\end{array}$ & $\begin{array}{l}59.6 \\
61.9\end{array}$ & 60 & 83 \\
\hline $\mathrm{Cdh} 3$ & $\begin{array}{l}\text { F, 5'-CTATTAGCGTCATCTCC-3' } \\
\text { R, 5'-CCTCGGCTGTTGTG-3' }\end{array}$ & $\begin{array}{l}52.2 \\
52.9\end{array}$ & 53 & 108 \\
\hline GAPDH & $\begin{array}{l}\text { F, 5'-GCCTTCCGTGTTCCTA-3' } \\
\text { R, 5'-AGACAACCTGGTCCTCA-3' }\end{array}$ & $\begin{array}{l}54.1 \\
54.6\end{array}$ & 54 & 110 \\
\hline$\beta$-actin & $\begin{array}{l}\text { F, 5'-TCTGTGTGGATTGGTGGCTCTA-3' } \\
\text { R, 5'-CTGCTTGCTGATCCACATCTG-3' }\end{array}$ & $\begin{array}{l}60.1 \\
59.8\end{array}$ & 60 & 135 \\
\hline
\end{tabular}

F, forward primer; $R$, reverse primer.

and pathway category results, four cell signaling transduction pathways were selected for verification analysis, including the cytokine-cytokine receptor interaction, nitrogen metabolism, coagulation cascades and cell adherens. Among these signaling pathways, Cxcl12, Tgfbr1, Asns, Bdkrb1 and Cdh3 genes were randomly selected and further verified by reverse transcription-quantitative polymerase chain reaction (RT-qPCR). The primers used in RT-qPCR were designed with the Premier 5.0 software and confirmed using Basic Local Alignment Search Tool analysis. GAPDH and $\beta$-actin were used as the internal control genes for the ED rat model and cultured CECs, respectively (Table I). cDNA was obtained using a RevertAid First Strand CDNA Synthesis kit (Fermentas, Waltham, MA, USA) for reverse transcription and a reverse transcription instrument (Mastercycler Gradient; Eppendorf, Hauppauge, NY, USA). Next, $20 \mu \mathrm{l} \mathrm{SYBR}{ }^{\circledR}$ Premix Ex Taq (Takara Bio, Inc., Otsu, Japan) was added and RT-qPCR was performed using a Realplex Mastercycler Ep Gradient S (Eppendorf) as follows: initial step of $60 \mathrm{sec}$ at $95^{\circ} \mathrm{C}$, followed by 40 cycles of $5 \mathrm{sec}$ at $95^{\circ} \mathrm{C}$ and $15 \mathrm{sec}$ at annealing temperatures (Table I). The relative expression levels of targeted genes were normalized to the expression levels of the internal control genes.

Statistical analysis. All the experimental data are presented as the mean \pm standard deviation. Student's t-test was used to analyze the differences between the groups. In addition, the differentially expressed genes of the treated CECs compared with the normal control cells were summarized (fold-change, $<0.5$ or $>2.0$ ). Analysis of the summarized oxidative injury-associated gene information was then performed for the various signaling pathways using SAS 3.0 software and differences in the results were identified using the categorized signaling pathways in the gene ontology (GO) and KEGG analysis. For the analysis of the RT-qPCR results, the following equations were used: ${ }^{\Delta} \mathrm{Ct}=\mathrm{Ct}$ - internal control $(\mathrm{Cti})$, and ${ }^{\Delta \Delta} \mathrm{Ct}=$ rat model $\left({ }^{\Delta} \mathrm{Ct}\right)+$ normal control $\left({ }^{\Delta} \mathrm{Ct}\right)$. The relative gene expression levels were calculated using the $2^{-\Delta \Delta \mathrm{Ct}}$ method (19) and compared. $\mathrm{P}<0.05$ was considered to indicate a statistically significant difference.

\section{Results}

Identification of CECs and RNA quality. Differential staining techniques were used to identify CECs, determine their survival rates and purity, as well as confirm the induction of OS. As shown in Fig. 1A, trypan blue staining revealed that the morphology of CECs was favorable, while the survival rate was found to be $99 \%$. The purity of the cell samples was determined by the positive rate of anti-vWF and found to be $96 \%$ (Fig. 1B) compared with the negative control cells (Fig. 1C). In addition, the purity of CD31 cells was determined by flow cytometry and found to be $92.7 \%$ (data not shown). Furthermore, the integrity of RNA extracted from the CECs and the corpora cavernosa of ED rats was demonstrated by RIN values of $>0.7$. In addition, the optical density 260/280 ratio was found to be 1.9-2.05 for the CEC and rat groups.

Screening for differentially expressed genes. Out of the total genes tested (41012), the normal and treated groups of the primary cultured cells were found to express 13,090 (31.92\%) and $12,039(29.35 \%)$ of these genes, respectively. A fold-change of 2.0 was considered to indicate differentially expressed genes compared with the control group. A total of 2,480 genes were found to be differentially expressed between the $\mathrm{X} / \mathrm{XO}$-treated and normal control groups. In total, 1,454 genes were upregulated and 1,026 genes were 
A

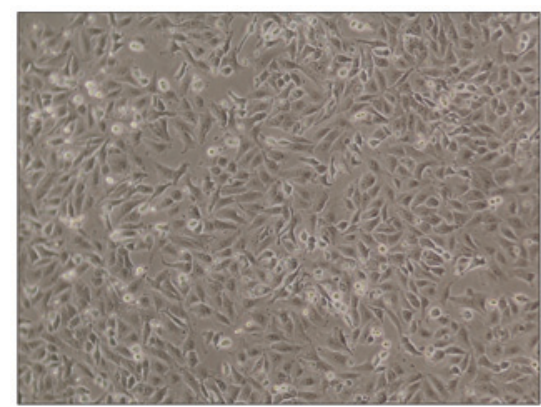

C

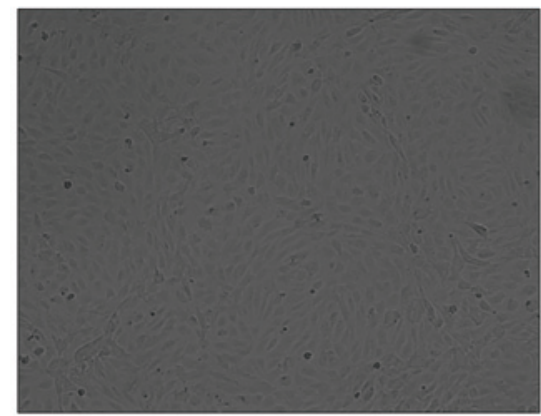

B

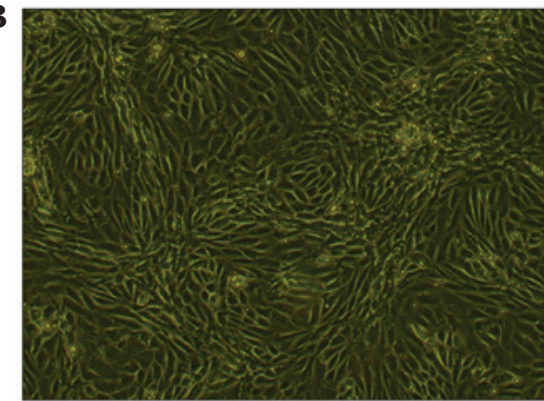

D

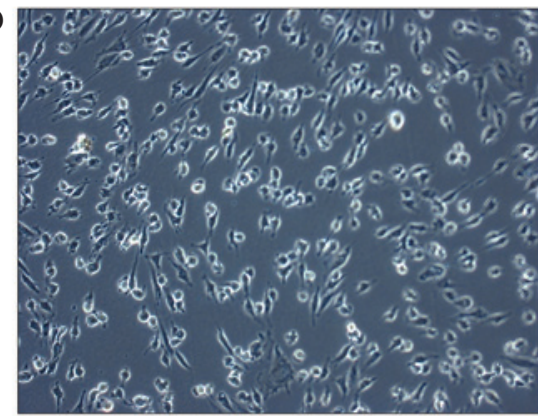

Figure 1. CEC identification, survival rates, purity and induction of oxidative stress. (A) Staining with trypan blue revealed that CECs exhibited a uniform fusiform appearance, with a spindle shape and good refraction ability, while the cell survival rate was 99\%. (B) Anti-von Willebrand factor immunofluorescence analysis was used to identify the purity of the separated and purified CECs. Positive staining rate was 96\%. (C) Negative control for purity analysis. (D) Following injury induced by exposure to xanthine/xanthine oxidase, the size and transparent ability of CECs were reduced compared with the normal control cells. CEC, cavernosal endothelial cells.

\section{A}

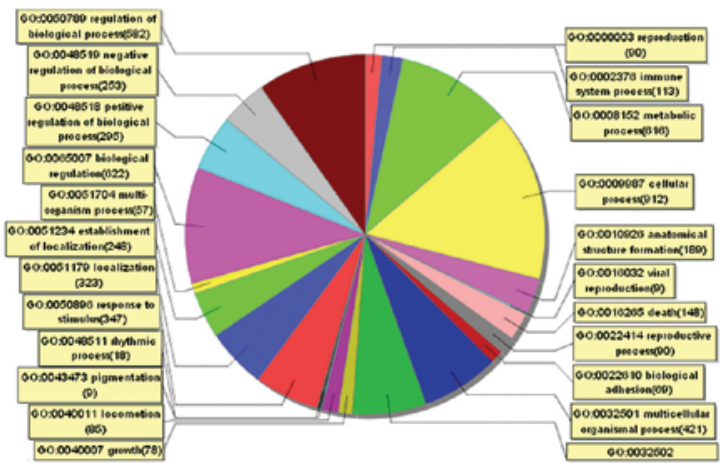

00:0000003 reproduction(90) • c0:00002376 immune system process(113) • c0:0008152 metabolic process:616] 00.0009987 cellular process $(912) \bullet 00.0010926$ anatomical stiucture foemation(189) $\bullet 00: 0016032$ vital reproduction(9)

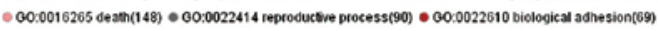

$\bullet$ 00:0032501 muliticellular organismal process(421) • 00:0032502 developmental process(395) • 00:0040007 growth(78)

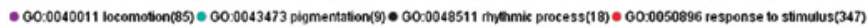

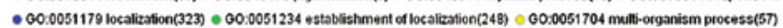

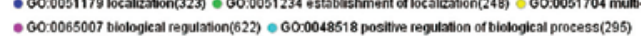

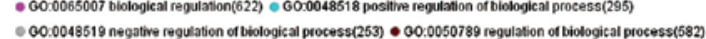

B

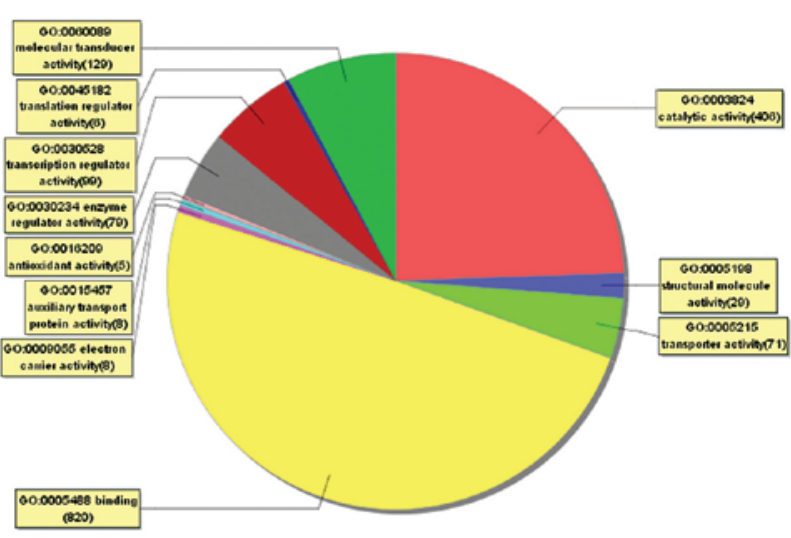

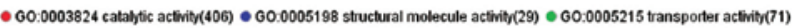

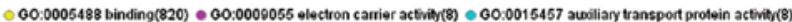

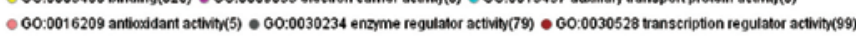
- G0:0045182 translaton regulator activity(6) • c0:0060089 molecular transducer activity(1 29)

\section{C}

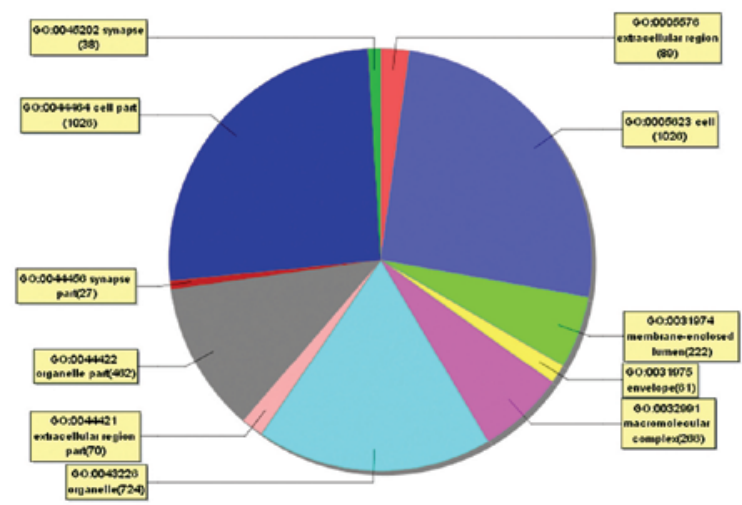

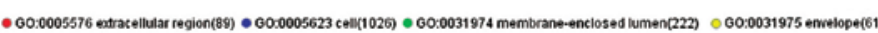

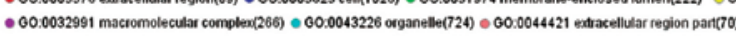

- c0:0044422 organelle par(462) • c0.00044456 synapse pant(27) • c0.0044464 cell part(1026) • c0:0045202 grapse(38)

Figure 2. Gene ontology analysis of the distribution of differentially expressed genes in categories of (A) biological process, (B) molecular function and (C) cellular component. 
Table II. The most downregulated and upregulated genes in the treated cavernosal endothelial cell group.

A, Downregulated genes

\begin{tabular}{|c|c|c|c|c|}
\hline Gene ID & Fold change & Symbol & Chromosome & Description \\
\hline 311311 & 0.0301 & Meis2 & 3 & Meis homeobox 2 \\
\hline 290409 & 0.0325 & Olfm4 & 15 & Olfactomedin 4 \\
\hline 292138 & 0.0327 & Eno4 & 1 & Similar to enolase (46.6 kD; 2J223) \\
\hline 310178 & 0.0328 & Myo10 & 2 & Myosin X \\
\hline 79430 & 0.0339 & Clcnkb & 5 & Chloride channel $\mathrm{Kb}$ \\
\hline 83579 & 0.0374 & Gnb5 & 8 & Guanine nucleotide binding protein, $\beta$-polypeptide 5 \\
\hline 83469 & 0.0378 & Lrp4 & 3 & Low density lipoprotein receptor-associated protein 4 \\
\hline 353227 & 0.0387 & Zbtb16 & 8 & Zinc finger and BTB domain containing 16 \\
\hline 302983 & 0.0409 & Mapk8ip3 & 10 & Mitogen-activated protein kinase 8 interacting protein 3 \\
\hline 362061 & 0.0479 & Cryz & 2 & Crystallin $\zeta$ \\
\hline 362911 & 0.0510 & $\mathrm{Mal} 2$ & 7 & Mal, T-cell differentiation protein 2 \\
\hline 290989 & 0.0559 & Catsper3 & 17 & Cation channel, sperm-associated 3 \\
\hline 315883 & 0.0582 & Plscr2 & 8 & Phospholipid scramblase 2 \\
\hline 685846 & 0.0587 & Adam34 & 16 & A disintegrin and metallopeptidase domain 34 \\
\hline 64896 & 0.0644 & Nolc1 & 1 & Nucleolar and coiled-body phosphoprotein 1 \\
\hline 309486 & 0.0650 & Tctn3 & 1 & Tectonic family member 3 \\
\hline 171576 & 0.0674 & Bub1b & 3 & $\begin{array}{l}\text { Budding uninhibited by benzimidazole } 1 \text { homolog mitotic check } \\
\text { point serine/threonine kinase B }\end{array}$ \\
\hline 302746 & 0.0700 & Mageb3 & $\mathrm{X}$ & Melanoma antigen family B, 3 \\
\hline 293808 & 0.0713 & Olr375 & 1 & Olfactory receptor 375 \\
\hline 309430 & 0.0714 & Dmrt2 & 1 & Doublesex and mab-3 associated transcription factor 2 \\
\hline 362867 & 0.0743 & Mybpc1 & 7 & Myosin binding protein $\mathrm{C}$, slow type \\
\hline 683313 & 0.0786 & LOC683313 & - & Similar to keratin complex 2 , basic, gene $6 a$ \\
\hline 360718 & 0.0807 & Popdc2 & 11 & Popeye domain-containing 2 \\
\hline 307545 & 0.0816 & Elp2 & 18 & Elongation protein 2 homolog (S.cerevisiae) \\
\hline 682105 & 0.0840 & Reep2 & - & Receptor accessory protein 2 \\
\hline 367012 & 0.0842 & Ddi1 & 8 & DNA-damage inducible 1 , homolog 1 (S. cerevisiae) \\
\hline 293897 & 0.0851 & Dkk1 & 1 & Dickkopf homolog 1 (Xenopus laevis) \\
\hline 365894 & 0.0887 & Trim33 & 2 & Tripartite motif-containing 33 \\
\hline 503269 & 0.0923 & Rab17 & 9 & RAB17, member RAS oncogene family \\
\hline 24772 & 0.1626 & $\mathrm{Cxcl} 12$ & 4 & Chemokine (C-X-C motif) ligand 12 (stromal cell-derived factor 1 ) \\
\hline 116777 & 0.2532 & Cdh3 & 19 & Cadherin 3 , type 1 , P-cadherin \\
\hline
\end{tabular}

B, Upregulated genes

\begin{tabular}{|c|c|c|c|c|}
\hline Gene ID & Fold change & Symbol & Chromosome & Description \\
\hline 85430 & 26.6004 & Herpud 1 & 19 & $\begin{array}{l}\text { Homocysteine-inducible, endoplasmic reticulum } \\
\text { stress-inducible, ubiquitin-like domain member } 1\end{array}$ \\
\hline 25617 & 24.0456 & Hspa5 & 3 & Heat shock protein 5 \\
\hline 25211 & 23.6140 & Lyz2 & 7 & Lysozyme 2 \\
\hline 501624 & 16.4694 & Bex4 & $\mathrm{X}$ & Brain expressed gene 4 \\
\hline 298961 & 15.4885 & Agr2 & 6 & Anterior gradient homolog 2 (Xenopus laevis) \\
\hline 295827 & 15.1051 & Olr597 & 3 & Olfactory receptor 597 \\
\hline 362196 & 15.0231 & Chac1 & 3 & ChaC, cation transport regulator homolog 1 \\
\hline 310738 & 14.6384 & $\mathrm{Ngf}$ & 2 & Nerve growth factor ( $\beta$ polypeptide $)$ \\
\hline 691491 & 13.8911 & LOC691491 & - & $\begin{array}{l}\text { Similar to Discs large homolog } 5 \text { (placenta and prostate DLG) } \\
\text { (discs large protein P-dlg) }\end{array}$ \\
\hline 304423 & 13.2743 & Tyw1 & 12 & tRNA-yW synthesizing protein 1 homolog (S. cerevisiae) \\
\hline 363288 & 13.2692 & Kif1a & 9 & Kinesin family member $1 \mathrm{~A}$ \\
\hline
\end{tabular}


Table II. Continued.

B, Continued

\begin{tabular}{|c|c|c|c|c|}
\hline Gene ID & Fold change & Symbol & Chromosome & Description \\
\hline 25059 & 13.1816 & $\mathrm{Hk} 2$ & 4 & Hexokinase 2 \\
\hline 65155 & 12.7013 & Alas1 & 8 & Aminolevulinate, $\delta-$, synthase 1 \\
\hline 685504 & 12.2517 & Clgn & 19 & Calmegin \\
\hline 83477 & 12.0425 & $\mathrm{Bcl} 10$ & 2 & B-cell CLL/lymphoma 10 \\
\hline 295750 & 11.6351 & Olr484 & 3 & Olfactory receptor 484 \\
\hline 307302 & 11.3526 & Cep120 & 18 & Centrosomal protein $120 \mathrm{kDa}$ \\
\hline 59322 & 10.8133 & Cnksr2 & $\mathrm{X}$ & Connector enhancer of kinase suppressor of Ras 2 \\
\hline 297417 & 10.1839 & Gfpt1 & 4 & Glutamine fructose-6-phosphate transaminase 1 \\
\hline 29393 & 10.1525 & Colla1 & 10 & Collagen, type I, $\alpha 1$ \\
\hline 59295 & 10.0863 & Nucb2 & 1 & Nucleobindin 2 \\
\hline 24908 & 10.0724 & Dnajb9 & 6 & DnaJ (Hsp40) homolog, subfamily B, member 9 \\
\hline 405265 & 10.0530 & Olr995 & 7 & Olfactory receptor 995 \\
\hline 362096 & 9.7722 & Setx & 3 & Senataxin \\
\hline 116595 & 9.5982 & Nrxn2 & 1 & Neurexin 2 \\
\hline 304376 & 9.5251 & Nyap1 & 12 & $\begin{array}{l}\text { Neuronal tyrosine-phosphorylated phosphoinositide- } \\
\text { 3-kinase adaptor } 1 \text {. }\end{array}$ \\
\hline 29215 & 9.3834 & Arg2 & 6 & Arginase type II \\
\hline 290997 & 9.3682 & Uimc1 & 17 & Ubiquitin interaction motif containing 1 \\
\hline 313255 & 9.3223 & Cip98 & 5 & CASK-interacting protein CIP98 \\
\hline 364755 & 9.0523 & Ero1lb & 17 & ERO1-like $\beta$ (S. cerevisiae) \\
\hline 29591 & 4.5276 & Tgfbr1 & 5 & Transforming growth factor, $\beta$ receptor 1 \\
\hline 25612 & 6.4865 & Asns & 4 & Asparagine synthetase \\
\hline 81509 & 2.7644 & Bdkrb1 & 6 & Bradykinin receptor $\mathrm{B} 1$ \\
\hline
\end{tabular}

downregulated in the $\mathrm{X} / \mathrm{XO}$-treated group. The detailed expression profiles of the most differentially expressed genes are illustrated in Table II.

Screening for signaling pathways in relevant databases. GO analysis was performed and the differentially expressed genes were further categorized according to biological processes, molecular functions and cellular components. In the differentially expressed genes associated with biological processes, the cellular and metabolic processes were found to account for $30.1 \%$. In the molecular function categorization, $54 \%$ of differentially expressed genes were associated with binding, particularly protein binding (72.3\%). In the analysis of cellular components, the cell part and cell were the most prevalent gene types (Fig. 2).

ED rat model establishment. ICP testing revealed a significantly lower peak in the ED rat model group $(3.21 \pm 1.20 \mathrm{mmHg})$ compared with the control rat group $(32.89 \pm 6.42 \mathrm{mmHg}$; $\mathrm{P}<0.05)$. Furthermore, the duration of erection was markedly shorter in the ED rats compared with the normal control rats, indicating that erectile dysfunction was induced in the ED rat model group.

Differentially expressed gene verification in vivo. The expression levels of Cxcl12, Tgfbr1, Asns, Bdkrb1 and Cdh3 genes

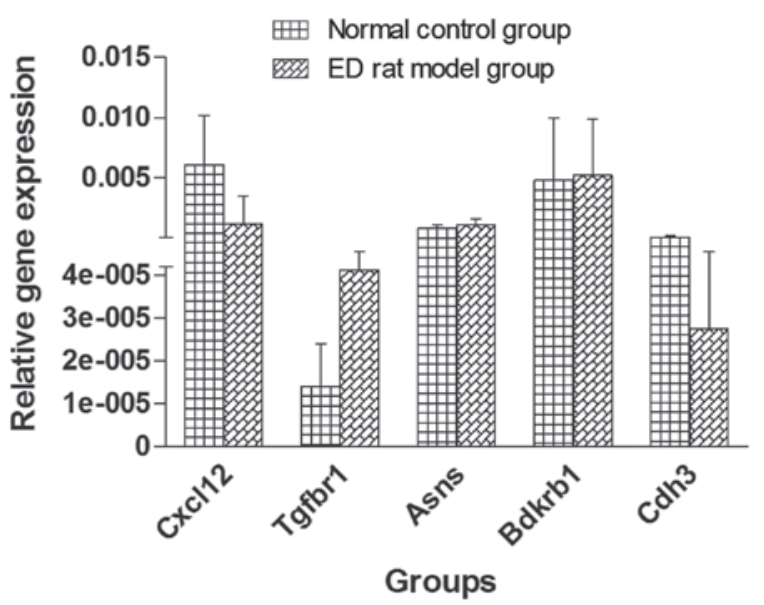

Figure 3. Reverse transcription-quantitative polymerase chain reaction was performed to determine the relative gene expression of five selected genes. Relative gene expression levels in ED rat models compared with those of the normal control group, respectively, were as follows: Cxcl12, 0.00123 vs. 0.00606 ; and $\mathrm{Cdh} 3,0.0000275$ vs. 0.0000818 , were decreased; whereas Tgfbr1, 0.0000413 vs. 0.000014; Asns, 0.00113 vs. 0.000834; and Bdkrb1, 0.00519 vs. 0.00478 , were increased. Trends in genes expression were comparable to those of the gene microarrays. ED, erectile dysfunction.

were analyzed using RT-qPCR (Fig. 3). The results revealed that the gene expression levels of $\mathrm{Cxcl} 12$ and $\mathrm{Cdh} 3$ were significantly downregulated in the ED rat group compared with the normal 
control rat group (0.16- and 0.25-fold change, respectively; $\mathrm{P}=0.000294$ and $\mathrm{P}=0.0001$, respectively). By contrast, Tgfbr1 expression was significantly upregulated in the ED rat group (4.53-fold; $\mathrm{P}=0.028$ ). The gene expression levels of Asns and Bdkrb1 were slightly increased in the ED rat group; however, the increase was not statistically significant compared with the normal control rat group. All the variation trends were consistent with the variations observed in the microarray results.

\section{Discussion}

Artery injury or ligation, diabetes mellitus, smoking, dyslipidemia and hypertension are closely associated with ED and may also induce OS $(1,2)$. Previous studies have demonstrated that OS and endothelial dysfunction were independent risk factors in the development of ED; however, associations among these three factors had not been widely explored. The pathogenic effect of OS in CECs and the associated endothelial dysfunction have been hypothesized to result in subsequential ED.

The results of the present study indicated that OS may lead to endothelial dysfunction through the upregulation or downregulation of cell signaling pathways involved in the secretion of inflammatory factors, NO metabolism, coagulation cascades and the expression of cell-surface adhesion molecules.

The genes validated in the present study were found to be differentially expressed in the aforementioned pathways. Genes associated with inflammatory factor reactions included Cxcl12, which was downregulated (0.16-fold), and Tgfbr1, which was upregulated (4.53-fold). Farouk et al (20) performed a genome-wide association study, which suggested that $\mathrm{Cxcl} 12$ was a potential target for atherosclerosis, vascular thrombosis and other diseases associated to endothelial dysfunction. In addition, Ho et al (21) reported that increased levels of stromal cell-derived factor 1 restored endothelial progenitor cells and increased local blood flow and perfusion, which may improve the symptoms of peripheral vascular disease and facilitate the recovery of injured tissue (22). Sengupta et al (23) studied the gene expression network in diabetes mellitus and identified that Tgfbr1 was able to induce OS. In addition, the authors verified that Tgfbr1 interacted with $\beta$-catenin, which gave rise to endothelial dysfunction via the Smad pathway. In the present study, the NO metabolism-associated gene, Asns, was found to be upregulated (6.49-fold difference). Fujita et al (22) also found Asns to be upregulated in impaired oxidative energy metabolism in a study on mitochondrial DNA mutation associations with mitochondrial dysfunction. Furthermore, these authors verified that mutated mitochondrial DNA promoted Asns expression through enhancing ATF4 gene expression. In the present study, Bdkrb1, which is associated with coagulation cascades, was found to be upregulated (2.76-fold) in ED rats. Bachvarov et al (24) also demonstrated that Bdkrb1 was upregulated in response to tissue injury or inflammation (25), while Kakoki et al (26) found that Bdkrb1 played an important role in relieving DNA damage and apoptosis, as well as maintaining the morphology and function of the kidneys. The present study also identified that the Cdh3 gene, which is associated with adhesion molecules, was downregulated (0.25-fold) in ED rats. Faraldo et al (27) previously reported that Cdh3 participated in the regulation of cell growth and differentiation. In addition, Yagi et al (28) proposed that cadherin was essential in the calcium-dependent cell-cell adhesion membrane glycoprotein at the adhesion junction, which also implied that functional proteins may be affected if the gene was misregulated.

In the present study, the candidate genes were found to be closely associated with OS, while the signaling pathways that these genes participate in were also found to be intimately co-associated with the pathogenesis of OS. Four signaling pathways and their key genes were selected for further verification and the results supported the effect of these OS-associated signaling pathways in endothelial dysfunction, which were also found to be differentially regulated in ED rats.

The trends of the candidate genes selected were consistent with the microarray analysis results; however, certain limitations are present in the current study. Bilateral internal iliac artery-ligation with hyperlipidemia may not fully reflect the morphological changes observed in the majority of ED patients, since morphological evidence from patients was not presented. In addition, various signaling pathways and differentially expressed genes were identified; however, only a minority of these were further investigated. Future functional verification is, therefore, required to confirm the conclusions of the present study and further elucidate the mechanisms of OS in ED.

In conclusion, the results of the present study identified and verified the dysregulation of OS-associated genes and signaling pathways in CECs and rats. These results indicated that the mechanisms of OS-induced endothelial dysfunction may proceed via the regulation of the identified genes and signaling pathways, which may also be involved in the development of ED. Further functional verification is required in order to elucidate the underlying mechanisms and signaling pathways associated with OS in ED.

\section{Acknowledgements}

This study was supported by major scientific and technological issues from the Science and Technology Commission of Shanghai Municipality, P.R. China (grant no. 08411951800).

\section{References}

1. Barrett-Connor E: Heart disease risk factors predict erectile dysfunction 25 years later (the Rancho Bernardo Study). Am J Cardiol 96: 3M-7M, 2005.

2. Mulhall J, Teloken P, Brock G and Kim E: Obesity, dyslipidemias and erectile dysfunction: a report of a subcommittee of the sexual medicine society of North America. J Sex Med 3: 778-786, 2006.

3. Zhang Q, Radisavljevic ZM, Siroky MB and Azadzoi KM: Dietary antioxidants improve arteriogenic erectile dysfunction. Int J Androl 34: 225-235, 2011.

4. Costa C and Virag R: The endothelial-erectile dysfunction connection: an essential update. J Sex Med 6: 2390-2404, 2009.

5. Musicki B, Liu T, Lagoda GA, et al: Hypercholesterolemia induced erectile dysfunction: endothelial nitric oxide synthase (eNOS) uncoupling in the mouse penis by NAD (P)H oxidase. J Sex Med 7: 3023-3032, 2010.

6. Morano S, Gatti A, Mandosi E, et al: Circulating monocyte oxidative activity is increased in patients with type 2 diabetes and erectile dysfunction. J Urol 177: 655-659, 2007.

7. Azadzoi KM, Golabek T, Radisavljevic ZM, Yalla SV and Siroky MB: Oxidative stress and neurodegeneration in penile ischaemia. BJU Int 105: 404-410, 2010.

8. Sullivan CJ, Teal TH, Luttrell IP, Tran KB, Peters MA and Wessells H: Microarray analysis reveals novel gene expression changes associated with erectile dysfunction in diabetic rats. Physiol Genomics 23: 192-205, 2005. 
9. Tusher VG, Tibshirani R and Chu G: Significance analysis of microarrays applied to the ionizing radiation response. Proc Natl Acad Sci USA 98: 5116-5121, 2001.

10. Agresti A: A survey of exact inference for contingency tables. Stat Sci 7: 131-153, 1992.

11. Storey JD, Taylor JE and Siegmund D: Strong control, conservative point estimation and simultaneous conservative consistency of false discovery rates: a unified approach. J R Stat Soc Series B 66: 187-205, 2004.

12. Nakaya A, Katayama T, Itoh M, et al: KEGG OC: a large-scale automatic construction of taxonomy-based ortholog clusters. Nucleic Acids Res 41: D353-D357, 2013.

13. Huang DW, Sherman BT and Lempicki RA. Systematic and integrative analysis of large gene lists using DAVID Bioinformatics Resources. Nature Protoc 4: 44-57, 2009.

14. Abe Y, Hotta Y, Okumura K, Kataoka T, Maeda Y and Kimura K: Temporal changes in erectile function and endothelium-dependent relaxing response of corpus cavernosal smooth muscle after ischemia by ligation of bilateral internal iliac arteries in the rabbit. J Pharmacol Sci 120: 250-253, 2012.

15. Lee MC, El-Sakka AI, Graziottin TM, Ho HC, Lin CS and Lue TF: The effect of vascular endothelial growth factor on a rat model of traumatic arteriogenic erectile dysfunction. J Urol 167: 761-767, 2002.

16. Albersen M, Fandel TM, Zhang H, et al: Pentoxifylline promotes recovery of erectile function in a rat model of postprostatectomy erectile dysfunction. Eur Urol 59: 286-296, 2011.

17. Wang J, Wang Q, Liu B, Li D, Yuan Z and Zhang H: A Chinese herbal formula, Shuganyiyang capsule, improves erectile function in male rats by modulating Nos-CGMP mediators. Urology 79: 241.e1-241.e6, 2012.

18. Albersen M, Lin G, Fandel TM, et al: Functional, metabolic, and morphologic characteristics of a novel rat model of type 2 diabetes-associated erectile dysfunction. Urology 78 : 476.e1-476.e8, 2011
19. Livak KJ and Schmittgen TD: Analysis of relative gene expression data using real-time quantitative PCR and the 2 (-Delta Delta C(T)) method. Methods 25: 402-408, 2001

20. Farouk SS, Rader DJ, Reilly MP and Mehta NN: CXCL12: a new player in coronary disease identified through human genetics. Trends Cardiovasc Med 20: 204-209, 2010.

21. Ho TK, Shiwen X, Abraham D, Tsui J and Baker D: Stromal-cell-derived factor-1 (SDF-1)/CXCL12 as potential target of therapeutic angiogenesis in critical leg ischaemia. Cardiol Res Pract 2012: 143209, 2012.

22. Lau TT and Wang DA: Stromal cell-derived factor-1 (SDF-1): homing factor for engineered regenerative medicine. Expert Opin Biol Ther 11: 189-197, 2011.

23. Sengupta U,Ukil S,DimitrovaN and Agrawal S: Expression-based network biology identifies alteration in key regulatory pathways of type 2 diabetes and associated risk/complications. PLoS One 4: e8100, 2009.

24. Fujita Y, Ito M, Nozawa Y, Yoneda M, Oshida Y and Tanaka M: CHOP (C/EBP homologous protein) and ASNS (asparagine synthetase) induction in cybrid cells harboring MELAS and NARP mitochondrial DNA mutations. Mitochondrion 7: 80-88, 2007.

25. Bachvarov DR, Hess JF, Menke JG, Larrivée JF and Marceau F: Structure and genomic organization of the human $\mathrm{B} 1$ receptor gene for kinins (BDKRB1). Genomics 33: 374-381, 1996.

26. Kakoki M, McGarrah RW, Kim HS and Smithies O: Bradykinin $\mathrm{B} 1$ and $\mathrm{B} 2$ receptors both have protective roles in renal ischemia/reperfusion injury. Proc Natl Acad Sci USA 104: 7576-7581, 2007.

27. Faraldo MM, Teulière J, Deugnier MA, et al: beta-Catenin regulates P-cadherin expression in mammary basal epithelial cells. FEBS Lett 581: 831-836, 2007.

28. Yagi T and Takeichi M: Cadherin superfamily genes: functions, genomic organization, and neurologic diversity. Genes Dev 14 1169-1180, 2000. 